ACCEPTED MANUSCRIPT

\title{
Chirality in fluorescence and energy transfer
}

To cite this article before publication: David L Andrews 2019 Methods Appl. Fluoresc. in press https://doi.org/10.1088/2050-6120/ab10f0

\section{Manuscript version: Accepted Manuscript}

Accepted Manuscript is "the version of the article accepted for publication including all changes made as a result of the peer review process, and which may also include the addition to the article by IOP Publishing of a header, an article ID, a cover sheet and/or an 'Accepted Manuscript' watermark, but excluding any other editing, typesetting or other changes made by IOP Publishing and/or its licensors"

This Accepted Manuscript is @ 2018 IOP Publishing Ltd.

During the embargo period (the 12 month period from the publication of the Version of Record of this article), the Accepted Manuscript is fully protected by copyright and cannot be reused or reposted elsewhere.

As the Version of Record of this article is going to be / has been published on a subscription basis, this Accepted Manuscript is available for reuse under a CC BY-NC-ND 3.0 licence after the 12 month embargo period.

After the embargo period, everyone is permitted to use copy and redistribute this article for non-commercial purposes only, provided that they adhere to all the terms of the licence https://creativecommons.org/licences/by-nc-nd/3.0

Although reasonable endeavours have been taken to obtain all necessary permissions from third parties to include their copyrighted content within this article, their full citation and copyright line may not be present in this Accepted Manuscript version. Before using any content from this article, please refer to the Version of Record on IOPscience once published for full citation and copyright details, as permissions will likely be required. All third party content is fully copyright protected, unless specifically stated otherwise in the figure caption in the Version of Record.

View the article online for updates and enhancements. 
TOPICAL REVIEW

\title{
Chirality in fluorescence and energy transfer
}

\author{
David L. Andrews \\ School of Chemistry, University of East Anglia, \\ Norwich Research Park, Norwich NR4 7TJ, \\ United Kingdom
}

\begin{abstract}
Optical polarization features associated with the fundamental processes of molecular fluorescence and resonance energy transfer are in general studied with reference to plane polarizations. When any of the species involved is chiral, the associated emission processes may exhibit an element of circular polarization - a degree of optical helicity. Although usually a minor effect, some systems can exhibit a sizeable component of circularly polarized luminescence, whose helicity correlates with the enantiomeric form. In studies of multi-component systems, in which initial excitation of a donor species followed by energy transfer - leads to emission from an acceptor molecule, the handedness of both donor and acceptor may influence output circularity. In systems with an achiral acceptor, a degree of fluorescence circularity may be influenced by the handedness of a chiral donor, but this should not be construed in terms of 'conveying' chirality. Chiral molecules may also play a passive role by inducing helicity in the fluorescence from/achiral neighbours, and further tiers of complexity arise if the initial excitation is itself of circular polarization. In all such processes, symmetry principles play a major role in determining a sensitivity to molecular handedness, and their detailed consideration enables a range of new experimental procedures to be identified. Casting the fundamental theory in terms of formal photon-molecule couplings enables the quantum mechanisms involved in all such phenomena to be clearly resolved. The results provide fresh physical insights, and establish connections across a range of indirectly related chiroptical phenomena including induced circular dichroism.
\end{abstract}

\section{Contents}

1. Introduction

2. Principles of chiroptical interaction

3. Symmetry-based categorization

4. Physical mechanisms and photonics

5. The interpretation of chiral emission

6. Measurement issues

7. Chirality and energy transfer

8. Connections to other chiroptical effects

9. Conclusion

References 


\section{Introduction}

In the field of molecular fluorescence spectroscopy, highly valuable information can be derived from the study of optical polarization. The interpretation of spectral data in the characterization of molecular samples, and the use of time-resolved studies to deduce dynamical behaviour, may both benefit from the additional information content of polarization-resolved measurements - as for example in ultrafast pump-probe studies of rotational relaxation [1-3]. Nonetheless, since emission studies of this kind are most commonly associated with plane polarizations, there is no real provision to identify any features that could signify a specific sense of molecular chirality. Conversely, the spectroscopic realm of chiroptical phenomena (processes that do display sensitivity to the handedness of chiral systems) is dominated by a small handful of effects such as optical rotation, circular dichroism, and Raman optical activity [4, 5] - all of them primarily being used as methods of analytical speciation or material characterization. Most other chiroptical effects highlighted in the recent literature relate to a potential harnessing of differential optical forces [6-11] - although it has become evident that the extreme subtlety of such effects substantially undermines prospects for their utility in efficient enantiomer separation [12]. Such is the context for several new, fluorescencerelated developments reported in this article, focusing on circularly polarized luminescence (CPL). The emphasis is on spin-allowed emission, though in the present connection the broader term 'luminescence' appears almost as often in the literature.

Despite a surprising omission from some well-known textbooks on molecular emission spectroscopy, the exploration of chirality in the realm of fluorescence and energy transfer is now an area of considerable significance, interest and potential. Pioneering work by Riehl and Richardson three decades ago [13, 14] first drew a focus onto this distinctive feature of fluorescent emission from specifically chiral molecules: its capacity to exhibit a small degree of ellipticity, originating in a subtle difference between the individual rates of left- and right-handed photon emission. Earlier studies has already shown how the resolution of rotational sub-levels could reveal much larger degrees of circularity in the emission from simple molecules such as molecular iodine [15-19], but these effects were not dependent on, or indicative of, molecular chirality. With the subsequent development of more sensitive instrumentation to resolve the much smaller signals of chiral behaviour, the field has developed and grown in importance at a steadily increasing pace. Certainly, the subject has innate difficulty: most of the phenomena it concerns are exhibited in quantitatively elusive effects, whose essential simplicity may be hidden by theories of considerable complexity, and which demand experiments that present a daunting level of challenge.

The transfer of electronic energy, widely known as RET or FRET - resonance energy transfer, or Förster resonance energy transfer - is a process that does not explicitly involve the real photons that constitute conventional light. (In rigorous theory it is represented in terms of a transfer, between the donor and acceptor, of virtual photons 
[20-30]: these are unobservable quanta of electromagnetic radiation, whose propagation lifetime is so short that quantum uncertainty entirely dominates their character. Since the associated optical modes are only ever fleetingly populated with single photons, stimulated amplification of this process in gain media is not a viable option.) Nonetheless, light is almost always implicated in absorption by the energy donor, prior to RET, and fluorescence emission by the energy acceptor, subsequent to the transfer process. Specialized studies of fluorescence and energy transfer processes may engage circular polarizations, in experimental configurations and mechanisms from which a range of novel effects can be elicited. Each process entails an additional level of experimental variable, beyond those widely familiar in fluorescence and RET studies. Accordingly, it is possible to identify new control applications: more significant is that fact that the exercise of controlled variation in experimental conditions provides access to new dimensions of information, to characterize either pure or complex samples.

In the overview that follows, the intention is not to provide a comprehensive survey of the field, nor its recent development: for those, the reader is referred to one of many timely surveys [31]. The intention is to bring a different perspective to the subject, focusing on issues of symmetry, and identifying photophysical mechanisms in detail but without layering the text with pages of mathematics. Only a few simple equations are needed to interpret the experimental implications, and a newly developed adaptation of Feymnan diagrams is deployed to clarify the flow of excitation through a system. Most important is the fact that an appreciation of the underlying principles can deliver fresh insights and obviate physically unfounded speculation. Chemical examples are drawn upon, where appropriate, to illustrative these features. The focus is largely, though not exclusively, on the common case of liquid or solution samples, allowing for significant orientational correlation between each donor and counterpart acceptor as within a host molecular framework - which is the case with many fluorescent protein studies, for example. Oriented or surface systems also represent scope for investigation, beyond the scope of this review: see for example refs [32-37].

With a primary focus on samples composed of chiral molecules, the initial aim is to develop and exploit a symmetry-based analysis to identify a surprisingly extensive range of phenomena, many of them proving to offer new opportunities for fruitful study. The range extends from a fairly obvious and well-studied instance of circularly polarized fluorescence, in which chiral molecules of given enantiomeric form produce output whose intensity varies with the handedness of the resolved emission, through to some relatively unusual effects - some of which seem not yet to have been an object of experimental investigation. Section 2 therefore begins with a comprehensive symmetry analysis, providing a basis for a complete categorization of permissible chiroptical processes in Section 3. The key to developing a systematic formulation of mechanism for such effects is a consideration of the fundamental photonics. Accordingly, Section 4 identifies the physical mechanisms by means of which these processes operate, focusing especially on their involvement of electric and magnetic transition moments, 
and in the following Section, theory for the interpretation of results is then discussed. Section 6 briefly focuses on associated measurement issues, and the following Section addresses the question of whether, or in what circumstances, chirality might itself be considered a 'transferable' quantity in association with RET. Section 8 considers the broad scope for more exotic effects, including the possible involvement of structured laser beams. The concluding Section of the review provides a summary of key findings, and it outlines opportunities for further developments in the field of chiroptical fluorescence.

\section{Principles of chiroptical interaction}

To gain clarity, and to provide a comprehensive framework to identify the various kinds of chiroptical process that may arise, it is worth beginning with a look at the conditions and requirements that are imposed on any such process by the demands of fundamental symmetry. In so doing, the primary focus will be on spatial parity for which the signature of each component of matter, and each optical field, is determined by its behaviour under the simple symmetry operation of inversion, denoted in the Schoenflies system by the symbol $i$. There is usually little additional benefit to be gained by considering, alongside it, the time-inversion symmetry which in principle might play an equally important role: generally, the conclusions of entertaining temporal symmetry are the same as those we can draw from spatial symmetry [38, 39], while the consequences of the latter are commonly simpler to envisage. Circular polarizations, for example, prove to be even with respect to time inversion - because their associated propagation vector also changes sign when time is reversed - but more obviously they change sign on space inversion. However, in the present connection a more global consideration of time symmetry does prove to add value, as will be seen in the next Section. (Temporal symmetry may prove of more significance for systems with an unpaired electron spin whose wavefunctions are odd with respect to time reversal - but to accommodate such relatively less common cases in a generalized symmetry analysis would entail a disproportionate level of additional complication. In Section 7 we shall briefly encounter a case of exciton spin states, where unpaired electron spin does become significant).

\section{- Chiral matter}

First, there are some salient details to note, concerning the criteria for molecular chirality and its association with a defined handedness. The general symmetry principles governing molecular chirality have been usefully reviewed by Harris et al. [40]. It is important to be clear from the outset that the familiar rules for $3 \mathrm{D}$ chirality, which demand the absence of any rotation-reflection axes - rules that also preclude mirror or inversion symmetry - are specifically the requirements for observations of chirality in fluid media. (Two-dimensional and metasurface forms of chirality impose 
less restrictive, though equally rigorous criteria, and these have been discussed in another recent review) [39].

As is shown in Fig. 1, this means that a molecule of chiral form will equally differ from its mirror image, which signifies the standard 'informal' chemical definition, and from the result of performing an inversion operation in three-dimensional space. The behaviour with respect to either operation is equally applicable as a condition in application to regular fluids, where the free rotation of individual molecules has no physically significance on internal transitions.
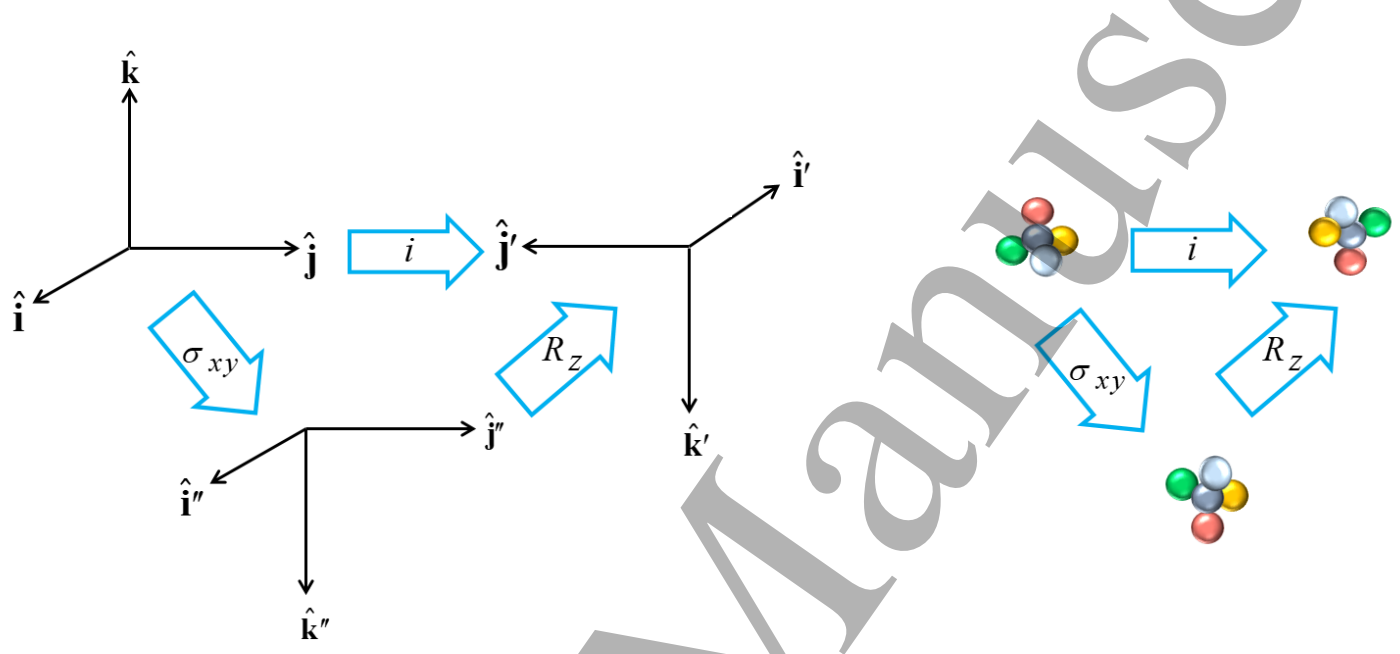

Figure 1. Symmetry transformations: on the left, passive operations on the coordinate frame; $\hat{\mathbf{i}}, \hat{\mathbf{j}}, \hat{\mathbf{k}}$ are unit vectors in the $x, y$ and $z$ directions, the former directed out of the page. On the right, active operations on prototypical molecules of opposite parity enantiomeric form. Each representation shows spatial inversion (top) as equivalent to mirror image rotation (the lower route in each case).

It is important to stress that for molecules there is no single absolute measure of intrinsic chirality. The familiar chemical designations of enantiomer specificity primarily facilitate an unequivocal attribution of oppositely handed forms. As is well known, the attribution of chirality to individual stereogenic centres does not account for all instances, though it simplifies the classification of molecules that do conform to this type. The fact that the chemical linkage of two oppositely handed moieties can give a product that is achiral also shows the importance of scale in assigning handedness. (It has been shown that local fields in the close proximity of even linear nano-antennas can exhibit chirality [41]). To accommodate all systems with intrinsic chirality, without restriction to those with any uniquely identifiable chiral centre, one must have recourse to the general definition. Most efforts to computationally identify molecular chirality 
have been based on the molecular geometry and associated symmetry [42, 43], or by modelling with the aid structure-activity-relationship principles [44, 45].

Nonetheless, for any given enantiomer, spectroscopic measures such as optical rotation and circular dichroism always exhibit dispersive behaviour, their measurements varying with the wavelength of light (as well as often exhibiting more obvious, chemical forms of dependence on temperature, concentration in solution or $\mathrm{pH}$, etc. The alternatively laevo- and dextro-rotatory behaviour of malic acid in this respect was first reported in 1930 [46]). For limited purposes it is possible to define a chirality pseudoscalar - a complex number with explicit dependence on optical frequency or wavelength (connected with optical rotatory dispersion) - but even for this it has been shown that both the real and imaginary parts necessarily exhibit a change in sign over certain ranges of frequency [47]. There are numerous examples in the literature of circular dichroic spectra exhibiting several variations in sign over even quite narrow ranges of wavelength; a classic illustration is the case of polyxanthylic acid reported by Eyring et al. [48]. Consequently - for the above reasons, amongst many others - it is evident that there can be no conserved chiral property, in the totality of the light and matter system, in any photon absorption or emission process [49]. The point is worth emphasizing since reports in the literature do occasionally, make such a claim.

For the purposes of clearly establishing symmetry criteria, it will prove expedient in the following analysis to assign to any pair of enantiomers a parameter $\xi= \pm 1$, where one sign belongs to each partner in the mirror-image pair. The choice of attribution is not important - its value lies only in serving as a discriminant though it could, for example, be chosen to correspond to the sign of standard optical rotation. (In this convention, however, $\xi=0$ is not an applicable representation for an achiral molecule.)

\section{- Chiral radiation}

For optical radiation, handedness is commonly associated with circular polarizations. Plane polarized light can be regarded as equivalent to an equal mix of left- and righthanded circular polarisations; elliptical polarisations tip the balance in favour of one handedness, while a pure circular polarization maximises the associated optical helicity. In contrast to the lack of any absolute measure of chirality for material systems, there is a scalar quantity that formally defines and exactly quantifies helicity for optical radiation [50]: for light with a finite linewidth, it can be defined in terms of a chirality density $\chi$ :

$$
\chi=\frac{1}{8 \pi^{3} c} \int k\left\{I^{(\mathrm{L})}(\mathbf{k})-I^{(\mathrm{R})}(\mathbf{k})\right\} d^{3} \mathbf{k},
$$


where $I^{(\mathrm{L})}(\mathbf{k})$ and $I^{(\mathrm{R})}(\mathbf{k})$ represent the intensities of left- and right-circularly polarized components for light of wave-vector $\mathbf{k}$ and frequency $\omega=c|\mathbf{k}|$, and other symbols have their usual meaning - with a caveat that $\chi$ is not the same as the angular parameter in Poincaré sphere representations of polarization. (The above expression is converted from quantum operator form [51] for application to radiation with a finite continuum of directions and frequencies.) The above expression in fact extends even to structured laser radiation, where another aspect of chirality may arise in connection with optical vortices (briefly to be discussed in Section 8); the above result retains its validity even for such beams.

The dependence on $k$ in equation (1) means that the formula has only approximate proportionality to the difference in left- and right-handed intensities, though the latter is an acceptable guide for light with a relatively narrow bandwidth. In this spirit, it is therefore once again helpful to introduce a parameter to indicate the oyerall handedness (for either optical input or output): let $\sigma= \pm 1$ specifically denote pure left-handed (upper sign) and pure right-handed (lower sign) circularly polarized states. This identification of sign is not arbitrary; it tallies with the sign of the chirality density, and it also correctly designates the quantity of spin angular momentum per circularly polarized photon, in units of the Dirac constant, $\hbar$. Here, $\sigma=0$ does correctly signify plane-polarized, or unpolarised radiation.

\section{Symmetry-based categorization}

Focusing now upon fluorescence and energy transfer processes, we can begin to exploit the parity signatures introduced in the previous Section, for each component of the process at the fundamental level, i.e. each photon and each molecule. Essentially, the symmetry product $\sigma \xi$ has to feature in one or more fundamental molecule-photon interactions, in order for some degree of chiroptical discrimination to be supported in any given process.

To begin with simple fluorescence, case (i) in the detailed descriptions that are to follow below, it will appear that there are indeed only these two system components to consider: the emitter and the emission. However, an indirect role may also be played by the optical radiation inducing initial electronic excitation, if it has a circular polarization; this is case (ii). This effect may, in principle, be manifest without regard to any degree of rotational or other form of relaxation prior to fluorescent emission; it simply reflects the potential for a marginally different rate of excitation (a circular dichroic effect) according to the relative handedness of the molecule and excitation radiation. So, in principle, there may be three relevant system components in case (ii), each with its own parity signature, determining the possibility and extent of any chiroptical features. For FRET, case (iii), the additional inclusion of a second material 
component (i.e. the acceptor) which itself may be chiral, raises the number of system components potentially involved to four.

In proceeding further, it will be useful to identify the number of combinations that give potentially different results - even where the differences will be only marginal. The number of distinct possibilities is, in each case, reduced by two principles: first, complete spatial inversion of the entire matter + radiation system cannot change the result; the second principle is that processes fundamentally linked by time-reversal should also have equivalent characteristics.

With regard to the first principle, it is to be noted that the relative handedness of the molecule and radiation needs to be considered independently for each photon-molecule interaction; it can be misleading to apply the notion of space inversion to the entirety of a process such as case (ii) above, since the absorption and emission are physically distinct processes. A caveat is also necessary in applying the second principle; due to the intramolecular vibrational relaxation that commonly intervenes between electronic excitation and emission - a feature that is, of course, reflected in a Stokes shift - to achieve an exact time-reversal of any absorption-emission sequence will in general be physically unrealistic. However, that is not an issue: for symmetry purposes, recognition of a difference between the input and output wavelengths is immaterial; it may be assumed that in any 'time-reversed' sequence, physically realistic wavelengths are deployed. To be clear, the intention here is to identify equivalences and differences between differential fluorescence signals - indicating the effects on sign, of a change in handedness, but not relating to absolute intensity.

\section{Simple fluorescence}

Table 1 presents the results for measurements of simple fluorescence, following electronic excitation with an unspecified (but commonly plane-polarized) beam. Each row represents a possible molecule-photon release combination, quantifiable in terms of a fluorescence signal intensity $F$. The handedness of the molecule is specified by $\xi$ and that of the emitted photon by $\sigma^{\prime}$; the product $\xi \sigma^{\prime}$ gives values that establish trivial identities between two pairs of measurement: $F_{4}=F_{1}$ and $F_{3}=F_{2}$. For a given specific enantiomer, only two possibilities arise. The fluorescence or emission dissymmetry factor, $g$, commonly defined as [52]:

$$
g=\frac{I_{\mathrm{L}}-I_{\mathrm{R}}}{\frac{1}{2}\left(I_{\mathrm{L}}+I_{\mathrm{R}}\right)}
$$

in which the subscripts denote intensities of left- and right-handed polarization, thus produces the following formulae for each member of an enantiomeric molecular pair: 
$g_{\mathrm{L}}=\frac{F_{1}-F_{2}}{\frac{1}{2}\left(F_{1}+F_{2}\right)} ; \quad g_{\mathrm{R}}=\frac{F_{3}-F_{4}}{\frac{1}{2}\left(F_{3}+F_{4}\right)} \equiv-g_{\mathrm{L}}$.

In equations (3), $\mathrm{L}$ and $\mathrm{R}$ stand for the handedness of each enantiomer.

\begin{tabular}{|c|c|c|c|c|c|}
\hline Rate & & & $\xi$ & $\sigma^{\prime}$ & $\xi \sigma^{\prime}$ \\
\hline$F_{1}$ & $\mathrm{~L}$ & $\mathrm{~L}$ & 1 & 1 & 1 \\
\hline$F_{2}$ & $\mathrm{~L}$ & $\mathrm{R}$ & 1 & -1 & -1 \\
\hline$F_{3}$ & $\mathrm{R}$ & $\mathrm{L}$ & -1 & 1 & -1 \\
\hline$F_{4}$ & $\mathrm{R}$ & $\mathrm{R}$ & -1 & -1 & 1 \\
\hline
\end{tabular}

Table 1. Parity signatures and discriminant (last column) for a chiral molecule emitting a circularly polarized photon

\section{(ii) Circularly excited fluorescence}

The number of possible symmetry combinations obviously increases when circularly polarized light of either handedness is deployed for the initial electronic excitation, as shown in Table 2. (The interplay of three independently chiral entities has also been shown to give rise to a similarly intricate set of possibilities for circular dichroism with optical vortex light [53]). In the present case, with $\sigma$ denoting the handedness of the excitation beam, there are three values for a discriminant now given by $\sigma \xi+\xi \sigma^{\prime}$, exhibiting the equivalences $F_{11}=F_{9}=F_{8}=F_{6} ; F_{12}=F_{5}$ and $F_{10}=F_{7}$. For example, the equality $F_{9}=F_{6}$ reflects a time-reversal symmetry.

Some of the differences in outcome may arise even for achiral samples, for which we may still observe $F_{6} \neq F_{5}, F_{8} \neq F_{7}, F_{10} \neq F_{9}$ and $F_{12} \neq F_{11}$. Accordingly, those configurations yielding a zero in the discriminant column can be discarded as lacking utility for chiral speciation. However, comparative measurements based on any of the other pairs with dissimilar discriminant values can give a chirally meaningful result. Thus we have, for example, the relation $F_{5}-F_{7}=F_{5}-F_{10}$ : the former equality designates a difference between the responses of opposite enantiomers, and the latter registers the circular differential response of one enantiomer. (As noted earlier, putting 
$\xi=0$ does not deliver the result for an achiral molecule - because these tables are predicated for use in connection with chiral matter. Depending on the angle of signal collection, even passive phenomena such as Rayleigh scattering can give differing results for ' $\mathrm{L}$ in $\rightarrow \mathrm{L}$ out' and ' $\mathrm{L}$ in $\rightarrow \mathrm{R}$ out' in achiral media: see Section 5).

\begin{tabular}{|c|c|c|c|c|c|c|c|}
\hline \multirow{2}{*}{$\begin{array}{l}\text { Rate } \\
F_{5}\end{array}$} & \multicolumn{4}{|c|}{ nnownonic } & $\xi$ & $\sigma^{\prime}$ & \\
\hline & $\mathrm{L}$ & $\mathrm{L}$ & $\mathrm{L}$ & 1 & 1 & 1 & \\
\hline$F_{6}$ & $\mathrm{~L}$ & $\mathrm{~L}$ & $\mathrm{R}$ & 1 & 1 & -1 & 0 \\
\hline$F_{7}$ & $\mathrm{~L}$ & $\mathrm{R}$ & $\mathrm{L}$ & 1 & -1 & 1 & -2 \\
\hline$\overline{F_{8}}$ & $\mathrm{~L}$ & $\mathrm{R}$ & $\mathrm{R}$ & 1 & -1 & -1 & 0 \\
\hline$F_{9}$ & $\mathrm{R}$ & $\mathrm{L}$ & $\mathrm{L}$ & - & & 1 & 0 \\
\hline$F_{10}$ & $\mathrm{R}$ & $\mathrm{L}$ & $\mathrm{R}$ & & 1 & -1 & -2 \\
\hline$F_{11}$ & $\mathrm{R}$ & $\mathrm{R}$ & $\mathrm{L}$ & & -1 & 1 & 0 \\
\hline$F_{12}$ & $\mathrm{R}$ & $\mathrm{R}$ & $\mathrm{R}$ & & -1 & -1 & 2 \\
\hline
\end{tabular}

Table 2. Fluorescence signatures and discriminant (last column) for a chiral molecule emitting a circularly polarized photon, following circularly polarized excitation (depicted colours of input and output for identification only)

Before moving on, it is additionally worth noting that there are some other trivial identities connecting the values in Table 2 with those for simple fluorescence measurements, given in Table 1. For example, for an unresolved or plane polarized input we have $F_{1}=1 / 2\left(F_{5}+F_{9}\right)$ etc.; the individual results are then readily shown to be consistent with setting $\sigma=0$ in Table 2. Indeed, it may be noted that averaging the discriminants in Table 2, for rows in which only the input polarization differs between $\mathrm{L}$ and R, correctly delivers the corresponding Table 1 results for an assumed planepolarized input.

(iii) Circularly excited FRET 
Allowing for the input absorption and output fluorescence to occur at different sites, coupled by RET, invokes a still higher level of complication - but some interesting new observations can be made. Here, the helicity signatures of the donor and acceptor are represented as $\xi_{\mathrm{D}}$ and $\xi_{\mathrm{A}}$, and it emerges that a measure of the relative handedness of each partner has to be incorporated in any definitive discriminant of potential outcomes. Including a factor that is the modulus of their difference proves the most expedient choice, and as shown in Table 3 it thus emerges that there are in principle six measurably different symmetry combinations, registered by discriminants in the range of integers $(-3, \ldots, 2)$.

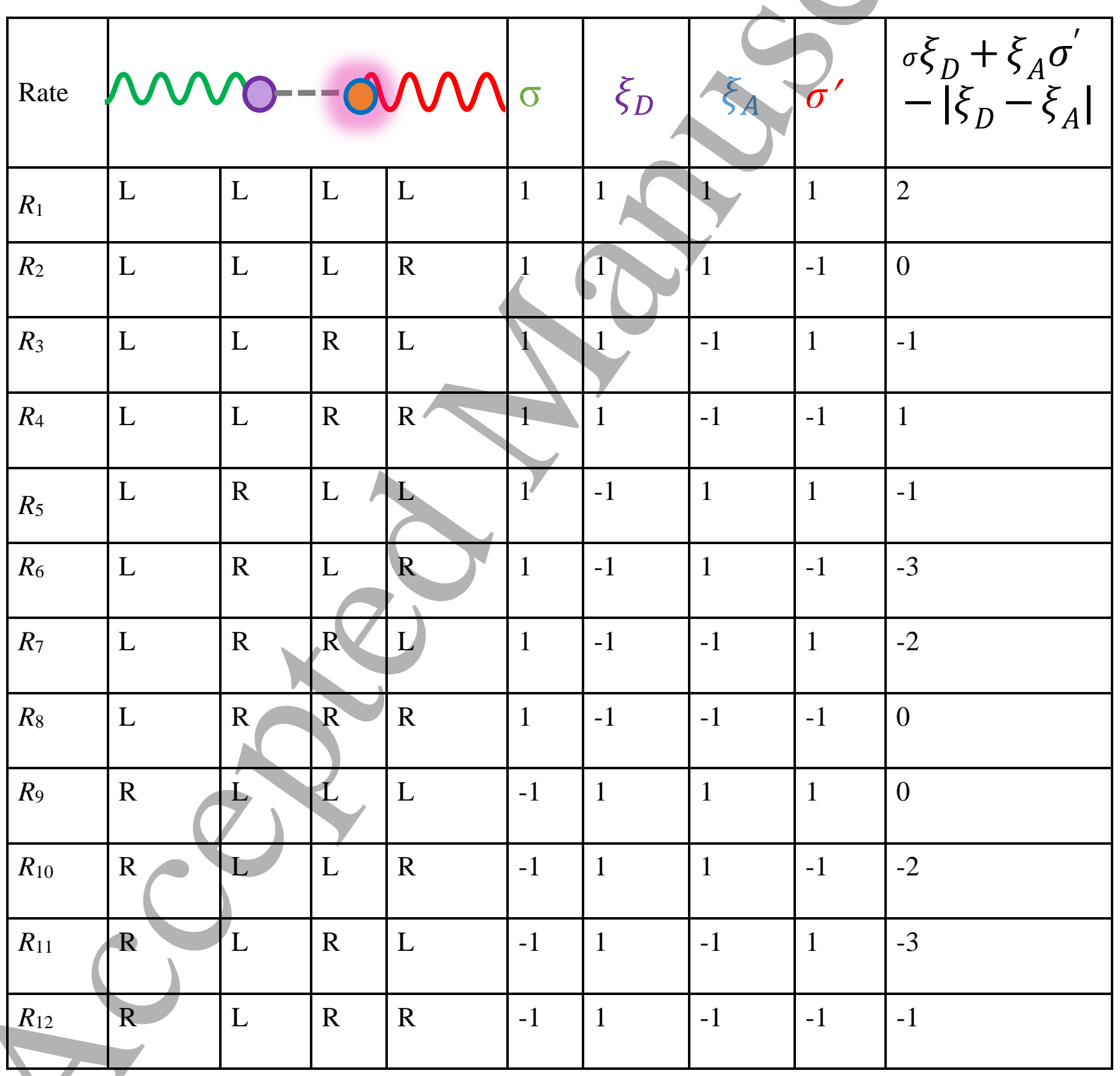




\begin{tabular}{|l|l|l|l|l|l|l|l|l|l|}
\hline$R_{13}$ & $\mathrm{R}$ & $\mathrm{R}$ & $\mathrm{L}$ & $\mathrm{L}$ & -1 & -1 & 1 & 1 & 1 \\
\hline$R_{14}$ & $\mathrm{R}$ & $\mathrm{R}$ & $\mathrm{L}$ & $\mathrm{R}$ & -1 & -1 & 1 & -1 & -1 \\
\hline$R_{15}$ & $\mathrm{R}$ & $\mathrm{R}$ & $\mathrm{R}$ & $\mathrm{L}$ & -1 & -1 & -1 & 1 & 0 \\
\hline$R_{16}$ & $\mathrm{R}$ & $\mathrm{R}$ & $\mathrm{R}$ & $\mathrm{R}$ & -1 & -1 & -1 & -1 & 2 \\
\hline
\end{tabular}

Table 3. FRET involving a pair of potentially chiral molecules, resolved for the emission of circular polarizations following circularly polarized excitation (depicted colours for identification only)

The large number of possible measurement pairs in this instance offers a multiplicity of routes to secure information on the chirality roles of donor and acceptor. As a simple example, suppose the donor is achiral but the acceptor is chiral and left-handed: with L input, the L-polarized fluorescence output from the acceptor is given by the summing of $R_{1}+R_{5}$, whose resultant discriminant value is 1 . Conversely, for ' $\mathrm{R}$ in and $\mathrm{R}$ out' the result is given by the sum $R_{10}+R_{14}$, and the corresponding discriminant sum is -3 . The individual numbers are unimportant - the sole significance is their being numerically different, from which it can be concluded that the fluorescence signal does indeed display a chiroptical effect: it will deliver different fluorescence intensities for the $\mathrm{L} \rightarrow \mathrm{L}$ and $\mathrm{R} \rightarrow \mathrm{R}$ configurations, duly reflecting the chirality of the acceptor. Analysing the range of symmetry combinations that may arise echoes the possibilities exhibited in optical binding $[10,54]$.

The results of this section serve to indicate which combinations of measurement can identify different aspects of chiroptical effect. The purpose of identifying discriminants is to label groups of study that must deliver essentially the same information; any pair of measurements used for chiral analysis should have different discriminant values. Clearly, there is a huge range of potential experiments with judiciously chosen combinations of input and output optical polarization. It is interesting to consider whether broad strategies for optical system design might be usefully informed by new methodologies for experimental procedural optimization [55].

\section{Physical mechanisms and photonics}

At the heart of each photon-molecule interaction, electrodynamic coupling occurs between the electric and magnetic fields of the radiation component, and the transition multipole moment properties of the molecule. The transition moments take various 
forms: the most significant are direct electric dipole (E1) coupling with the electric field, magnetic dipole (M1) coupling with the magnetic field, and electric quadrupole (E2) engaging with the local electric field gradient. For present purposes the detailed form of these interactions need not concern us, but details are provided in numerous textbook, review articles and papers - see for example ref [56]. For molecules in general, E1 transition couplings may typically be stronger than M1 or E2 by several orders of magnitude, but the different selection rules for each multipole, and for each individual electronic transition, determine whether each form of coupling is even allowed. In this respect a key property of chiral molecules, owing to the complete preclusion of rotationreflection axes noted earlier, (which means that such molecules belong to one of the 'pure rotation' symmetry point groups, $\mathrm{C}_{n}, \mathrm{D}_{n}, \mathrm{~T}, \mathrm{O}$ and I), is that for such species every E1-allowed transition is also M1-allowed, and in many cases it is additionally E2allowed.

At a fundamental level all three of the primary transition multipoles - E1, M1 and E2 are involved in common manifestations of chiroptical phenomena. (The involvement of E2 multipoles only disappears the expressions for optical rotation and circular dichroism in fluid media, due to the stochastic effects of molecular rotation). Often their quantitative transition moments for specific transitions are denoted by the use of superscripts as, for example, $\mu^{u 0}$ for electric dipole emission $|u\rangle \rightarrow|0\rangle$. For present purposes it will suffice to use the simpler form $\boldsymbol{\mu}^{\downarrow}$, with $\boldsymbol{m}^{\downarrow}$ and $\boldsymbol{Q}^{\downarrow}$ its M1 and E2 counterparts.

As illustrated schematically in Fig. 2, E1 moments have a polar vector nature whose character is formally of odd parity with respect to space inversion, whereas M1 (a pseudovector) and E2 (a second rank tensor) are even. For each of the three scenarios examined in Section 3, one can therefore entertain a sequence of successive transition interactions, in each stage in which - for a chiral molecule - any of these multipoles might play a contributory role. This is schematically indicated in Fig. 3, with regard to E1 and M1 alone; the coupling between donor and acceptor in the case of FRET is mediated by a dipole-dipole tensor interaction that is conventionally labelled $\boldsymbol{V}$ for E1E1 (equally M1-M1) or $\boldsymbol{U}$ for E1-M1 and M1-E1 [26, 27, 57]. Also (not shown) E2 couplings may occur. It is worth noting that the mechanism for all such couplings is fundamentally identical to near-field electrodynamical coupling. The full nature of these donor-acceptor couplings nonetheless repays further attention, and will be examined in more detail in Section 7.

Further insights into the mechanisms are afforded by the modified Feynman diagrams shown in Fig. 4, which in full implementation support a robust theoretical method to determine the quantum amplitudes that underpin the rate equations, as we shall see in the next Section. The nature and structure of all the coupling multipoles and tensors has been given in numerous other previous publications - see for example refs. [26, 58]. 


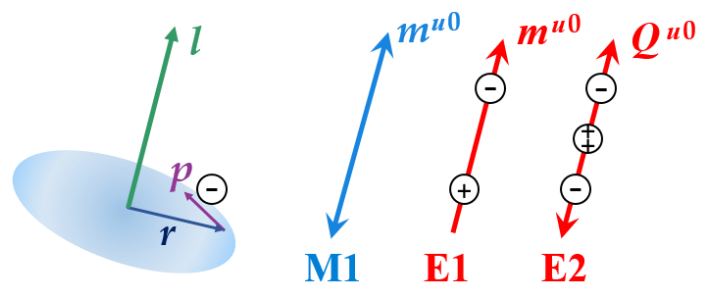

Figure 2. Representations of multipole symmetries. Left: the angular momentum (AM) of an orbiting electron, given by the vector cross-product of position and momentum $l$ $=\boldsymbol{r} \times \boldsymbol{p}$, is a pseudovector (even under space inversion) since $\boldsymbol{r}$ and $\boldsymbol{p}$ are polar vectors: hence M1 transition moments, based on electron AM, are even parity. Electric transition dipoles (E1) are odd, transition electric quadrupoles (E2) even. (The latter is not a vector but a tensor property; an 'edge-on' aspect is depicted to exhibit even character).

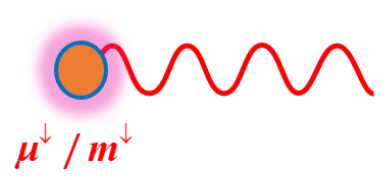

(i)

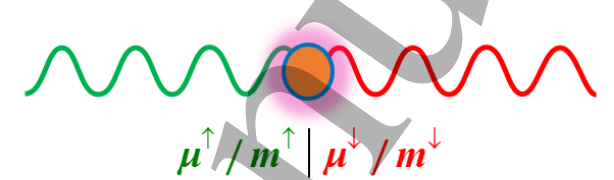

(ii)

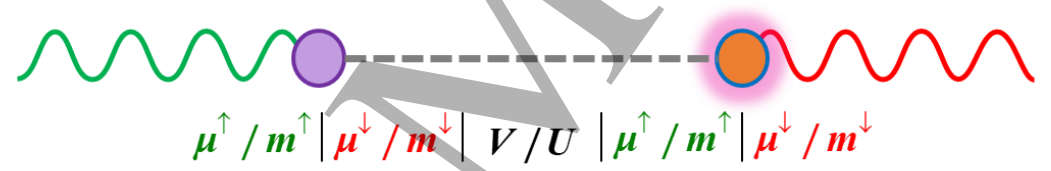

(iii)

Figure 3. (i) and (ii) Electric and magnetic transition dipoles ( $\boldsymbol{\mu}$ and $\boldsymbol{m}$ respectively, superscript arrows denoting excitation or decay) engaged with light in fluorescence studies; (iii) and also, for FRET, in an additional interaction between multipoles (dotted line). Colours here serye only to emphasize the distinction between multipoles involved in excitation (green) and those involved in relaxation/emission (red). In each multipole interaction, electric quadrupole and higher multipoles (not shown) may also participate. 


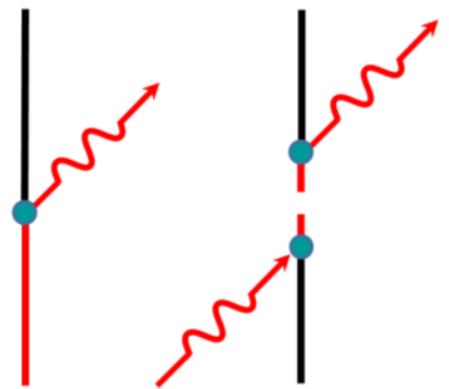

(i) (ii)

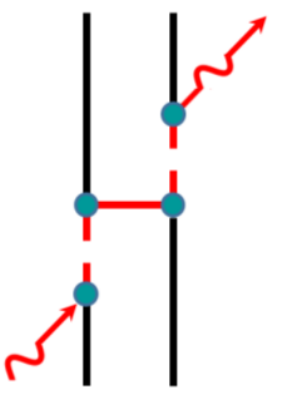

(iii)

Figure 4. Modified Feynman diagrams of typical form for each of the fluorescence processes delineated in Sections 3 and 4. Time progresses upwards, and in each case, the interaction sequence can be traced up through the red lines denoting energy flow. Solid vertical lines of red (black) represent excited (ground) states, broken lines indicating a finite lifetime electronic excited state typically entailing intramolecular vibrational relaxation; wavy lines denote input and output photons. (i) Direct fluorescence; (ii) Absorption followed by fluorescence; (iii) Absorption followed by RET and then fluorescence. Here the horizontal line represents RET coupling between the two centres (represented by two vertical world-lines), with the donor on the left and acceptor on the right. The dots indicate electrodynamic coupling, predominantly of E1, M1 or E2 character.

\section{The interpretation of chiral emission}

The intensity of emission, for any direct fluorescence or RET process, is determined by factors associated with each component in the sequence of fundamental interactions there are three, for example, in case (iii) of Section 3: donor absorption, RET and acceptor fluorescence. For each step, the quantum efficiency depends quadratically on a sum of contributions to a 'quantum amplitude' $M$, which has contributions from every combination of individually allowed electronic transition moments - see for example Section 7 in ref. [38]. In general, the photochemical rate constant $k_{\phi}$ for each component can be derived from Fermi's Golden Rule [59]:

$$
k_{\phi}=\frac{2 \pi \rho}{\hbar}|M|^{2}
$$

The density of states $\rho$ is formally a convolution of functions representing the number of states per unit energy interval for each of the light-matter system components; in practice, for chiroptical phenomena the linewidth of the molecular excited state alone usually determines the value. For example, in the convolution of the density of states for the input radiation and for the electronic excited state of any reasonably large 
polyatomic molecule, the sharper distribution of states determines the shape of the convolved energy distribution function. In spectroscopy with a typically narrow linewidth laser source, any excited state lifetime on the nanosecond or longer timescale will have a much narrower Fourier-transform frequency spread.

As described earlier, for each single-interaction process, $M$ thus comprises a simple sum of terms $M(\mathrm{E} 1)+M(\mathrm{M} 1)+M(\mathrm{E} 2)$. Thus, each of the scenarios examined in Section 3 entails a sequence of allowed transitions in each of which - for a chiral molecule - any of these multipoles might play a role. Thus interference terms arise, in the quadratic sum of contributions, between sequences with one or more differences in specific moments; it is these cross-terms that represent the potential for chiroptical differentiation. Fig. 5 shows, for example, relevant multipole interferences in the simple case of fluorescence (i), indicating their relative quantitative significance. It follows from equation (2) that the $g$ value is primarily determined by the following;

$$
g \sim 2 \operatorname{Re}\{M(\mathrm{E} 1) \bar{M}(\mathrm{M} 1)+M(\mathrm{E} 1) \bar{M}(\mathrm{E} 2)\} /|M(\mathrm{E} 1)|^{2},
$$

where both the numerator and denominator have higher order terms (not shown) that are almost entirely negligible. Clearly, the sign of $g$ is determined by the numerator of this expression, alone.

Figure 5. Half-table showing the symmetry character for the interferences of electric and magnetic transition dipoles in individual fluorescence interactions; for the fundamental transition multipoles green colouring denotes odd spatial parity, and red denotes even. At the intersections, purple identifies products of these multipoles that can display a sensitivity to chirality; the two interferences with paler purple colouring have this same character, but they are significantly weaker effects.

For multi-step processes, the same basic rules apply, and the overall rate constant conflates the quantum amplitudes $M$ for each individual step. In case (iii), for example, there can arise an interference between interaction sequences $\mu^{\uparrow} \mu^{\downarrow} V \mu^{\uparrow} \mu^{\downarrow}$ and 
$m^{\uparrow} \mu^{\downarrow} V \mu^{\uparrow} \mu^{\downarrow}$ where, as indicated in Fig. 2, the first pair of upward and downward transitions relate to the donor, and the second pair to the acceptor. For the reasons examined in earlier Sections, it is clear that all such interference terms involving products of opposite parity, for any particular interaction component, will arise only when the species at which the corresponding transition occurs, is a chiral centre (the donor in this particular example). Equally, the possible involvement of both $U$ and $V$ coupling tensors can support chiral discrimination in the process of excitation transfer [60-62].

Now, to apply the symmetry principles developed in Section 3, let us consider the case of intermediate complexity, case (ii), for which the possible experimental configurations and results are illustrated in Table 2. Suppose the initial electronic excitation is produced with a beam of left-handed circular polarization, and on resolving the emission (using a quarter-wave plate and linear polarizer, for example) a difference is found between the relative intensities of left- and right-handed components. Table 2 indicates that either $F_{5}$ and $F_{6}$, or $F_{7}$ and $F_{8}$, is responsible - either case proving the chiral nature of the fluorophore, though not its handedness. Again, it is important to note that absolute handedness is never resolvable - nor is it even a meaningful concept, unless in relation to other, known enantiomers. (For any set of structurally related compounds, any handedness label afforded by circular differential fluorescence measurements need have no correlation, even in sign, with the specific optical rotation for the same materials).

\section{Measurement issues}

Whilst it is not the purpose of this review to describe specific forms of instrumental methodology, it is important to be specific about collection of the fluorescence signal. The above conclusion is valid when the conventional right-angled detection geometry is employed for the measurements, with orthogonal directions for the input and detection. In fact, the validity extends to fluorescence intensity measurements integrated over all angles of emission, as might for example be achieved using an integrating sphere. But there is a caveat: for collection of the fluorescence at any other specific, limited angle, it is generally possible for the ' $L$ in $\rightarrow$ L out' and ' $L$ in $\rightarrow R$ out' experiments to give differing results even in the E1 approximation - which means that even achiral molecules may exhibit such an effect. (This relates to the fact that angular momentum conseryation applies when a system is invariant under rotation about the corresponding axis. Observation at right-angles, however, precludes the operation of this principle for the entire interaction sequence: in this respect the input and output events are entirely separable).

There are also other, more practical considerations. Although any beam of light with unequal populations of left- and right-handed circular polarizations can be represented 
in terms of ellipticity, this is not a feature amenable to direct measurement in fluorescence output. The reason is the generally random orientation of the ellipse unless the initial excitation were to be from a plane polarized beam and the fluorescence lifetime much less than the timescale for rotational relaxation. This is why expressions such as equation (2) are cast in terms of relative intensities of the two opposite circular polarizations. However, unlike orientational relaxation methods that deploy linear input polarizations to resolve rotational decay times, the subtle circular differential in the luminescence associated with circularly polarized input is not so substantially affected by electronic/vibronic and rotational decay.

Fluorescence detection of molecular chirality has the advantage over circular dichroism (CD) of being a zero-background method, although their shared dependence on weak M1 transition dipoles indicates that the circular differential is usually very small and experimentally challenging to measure. For example the degree of circular polarization in emission from substituted helicenes - molecules often serving an iconic role as representatives of chiral species - is typically $0.1 \%$ 463]. (The detection of any corresponding ellipticity would be exceptionally demanding). Securing robust measurements from simple organic molecules is sufficiently challenging by any means [64]. With more extended molecular systems, whose chirality can extend over a greater volume, substantially larger dissymmetry values have been achieved in several interesting cases; a notable recent example from Cerdán et al. [65] is their report on CPL from a BODIPY dye in limonene, whose spectra are shown in Fig. 6. Configuring the system to exhibit laser emission produced exceptional $g$ values in the range $0.1-$ 0.2 . This is not a trivial effect: in suitably confined systems, fluorescence radiation produced either directly, or indirectly through FRET, may itself be sufficiently intense to produce any degree of stimulated emission. The significance is simply that, by amplifying any initial imbalance between the relative numbers of left and right-handed photons, the effect has a capacity to give rise to an anomalously high circular intensity differential. 

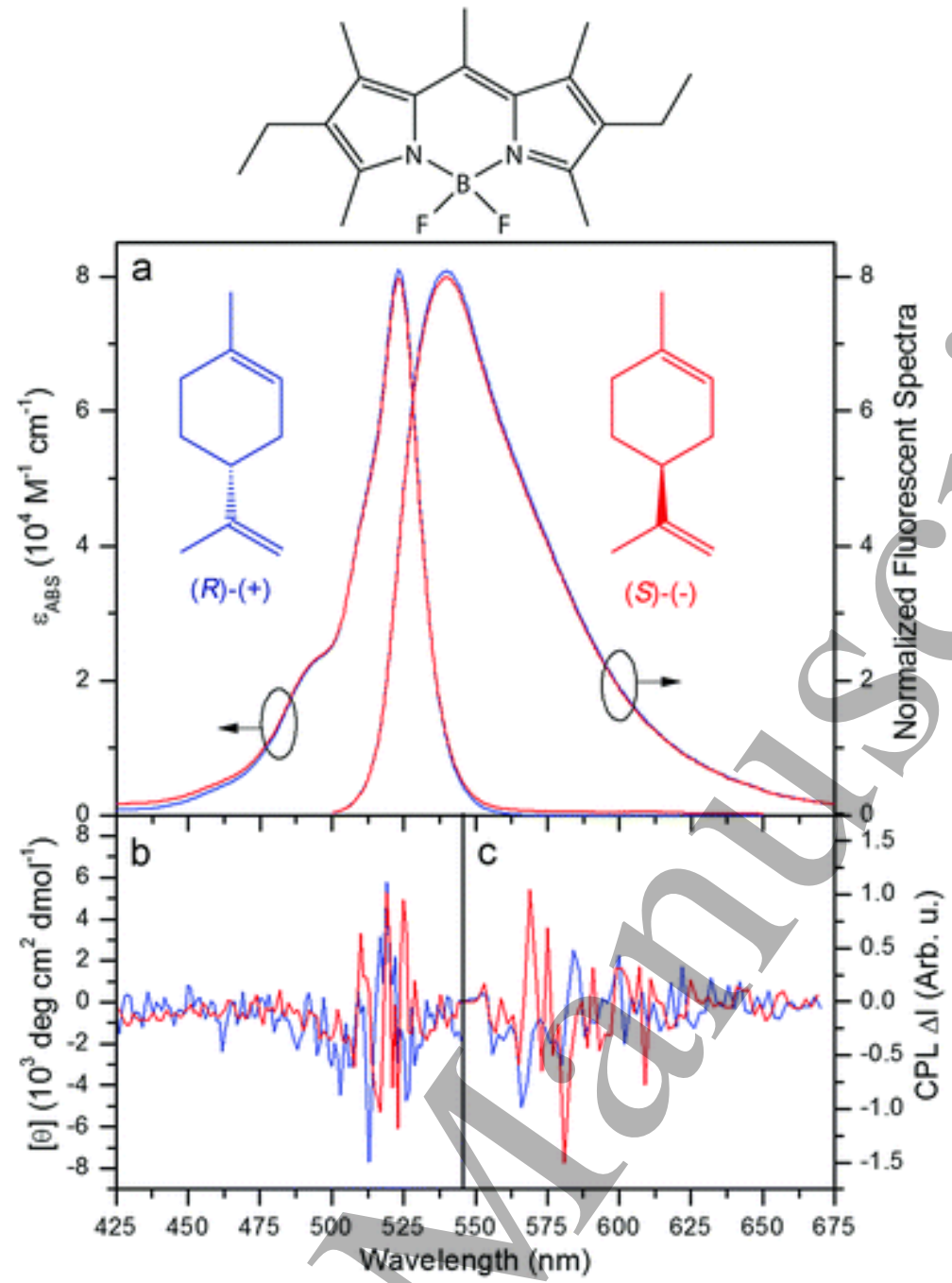

Figure 6. Spectra of the achiral BODIPY dye PM567 in (R)-(+)- and (S)-(-)-limonene (blue and red curves, respectively). (a) Extinction coefficient $\varepsilon_{A B S}$ and normalized fluorescence spectra, (b) CD molar ellipticity [ $\theta]$, and (c) CPL spectra. Inset and top: Chemical structures of dye PM567 and the two enantiomers of limonene. Reproduced by permission of the Royal Society of Chemistry from ref. [65].

Another aspect of measurement worth noting is the distinctive form of fluorescence measurements made in the close proximity of the emitter. It has been shown that, whereas for a strong field the circular dissymmetry would in principle be given by a ratio of Stokes parameters, $g=-S_{3} / S_{0}$, extension of the same operator formalism into the near-field fails to deliver an accurate representation [66]. In particular, a substantial change in polarization behaviour occurs within detection distances typically ranging out to about $0.3 \mu \mathrm{m}-\mathrm{a}$ few reduced wavelengths $\lambda$, where $\lambda=\lambda / 2 \pi$ and $\lambda$ is the wavelength of the emission. Fluorescence anisotropy, for example can also vary dramatically over this interval [67]. Calculations on the degree of circularity in fluorescence emission from surface functionalized (surface-coated) nanoparticles 
reveal a striking change between measurements in the near-field region $(\lambda R<1)$ and the more common far-field $(\lambda R>1)$ observed with a remote detector [68].

It should be emphasized that in the increasingly popular study of fluorescence and associated FRET processes in highly complex media, such as those comprising fluorescence proteins [69], heterogeneity can often arise in the initially excited electronic state population. To disentangle the signals from different components calls for cleverly designed investigative procedures [70-72], ideally combining spectroscopic and temporal resolution. It may be anticipated that the resolution of chiral features in any such system will present additional challenge - yet the prize will be an additional tier of information concerning the local symmetry attributes of the relevant state components.

\section{Chirality and energy transfer}

Recently, a small group of authors have entertained the proposition that energy transfer may be accompanied by 'chirality transfer' [73-75]. Such a notion has superficial appeal, although caveats have already been given concerning the difficulty in absolutely quantifying molecular chirality. It may also be observed that, in the simpler process of direct excitation using a circularly polarized beam - equally by capture of a single photon emitted by a remote chiral source - there is no sense in which the absorption of a photon either reduces or increases the chirality of the absorber. However, to fully address the substance of the specific claim it is necessary to take recourse to a more fundamental theory. In order to develop a correct electrodynamical representation, and to provide clear grounds to redress a number of misinterpretations that have recently appeared at various places in the literature, it will be helpful to briefly recap on the definitive quantum theory of RET.

It is now well known that, beyond the region of significant overlap of wavefunctions between any electronically excited donor and counterpart acceptor molecule in its ground state, RET proceeds by a near-field coupling mechanism. In a classical cast of theory, this coupling is understood as a retarded interaction between oscillating electric dipoles $[76,77]$ : in the quantum representation that is truer to the nature of molecular transitions, the coupling is represented in terms of a mechanism in which virtual photons convey excitation energy between donor and acceptor transition dipoles [20-30]. It is these formulations that lead to the explicit forms of the $\boldsymbol{U}$ and $\boldsymbol{V}$ coupling tensors [26, 27, 57]: in fact, both the classical and quantum representations deliver the same analytical results.

Adopting the fully quantum perspective, theory demands that the wave-vectors and polarizations of the virtual photons are summed over an unlimited range - consistent with Feynman's path-integral formulation. In particular, polarization aspects can be 
cast as a sum over any pair of polarizations with mutually orthogonal polarization vectors. Although perpendicular plane polarizations are often deployed in this summation, it is equally possible to choose left- and right-handed circular polarizations, and it is this latter choice that better suits our present purposes. As mentioned earlier, a discrete unit of spin angular momentum is associated with each circular polarization. (Circular polarization states are the only states of a photon that convey well-defined values of both energy and angular momentum [78]). In consequence, it emerges that, through the mediation of one circular form, it is possible to conserve angular momentum in the course of energy transfer. Moreover, since the selection rules for the absorption of circularly polarized light provide for exciton spin states to be optically oriented, energy migration along a chain of quantum dots with common orientation can preserve spin information with complete fidelity [79], a feature that has an important role in developing optically programmable spin memory [80-82].

For materials with closed shell electron configurations, however, there is no connection through which angular momentum can be conveyed through the process of resonance energy transfer, as has been proven in a detailed multipolar analysis [83]. This has added significance since, as is widely known, helicity can only be conveyed in electrodynamic form through association with angular momentum [84]. Moreover, the quantization of that angular momentum is only valid along a single axis, which identifies with the propagation vector. Therefore the prospect of helicity transfer in fluorescence RET $[85,86]$ is not supported by fundamental principles. A cited appeal by the latter group to a definitive treatise on quantum optics [87] is misjudged: its treatment of theory focuses upon atoms (whose spherical symmetry supports the correlation of photon spin with the Laporte selection rules, for example). The lack of such symmetry in a molecule - especially a chiral molecule - thoroughly undermines the notion of a supported 'rotating dipole state' with defined helicity.

Furthermore, it is to be borne in mind that the experimentally significant timescale for RET, associated with a rate constant that is measured for bulk media, quantifies the simplest measure of a stochastic temporal distribution of transfer events - whereas each individual transfer event occurs on the sub-femtosecond timescale of virtual photon propagation between the donor and acceptor. (Indeed it is because the propagation time is so short that it so strongly manifests the operation of the time-energy uncertainty principle, inextricably linked with the characteristic inverse sixth-power dependence on distance [26]). In summary, therefore, nothing measurably significant occurs during the ultrashort interval of any individual energy transfer event, nor can helicity transfer accompany it.

There are, however, features associated with chiral energy transfer that, it seems, have not before received adequate attention. Consider, for example, the inverse sixth power dependence on distance, specifically associated with short-range (Förster) energy transfer. As is well known, this originates from a quadratric dependence of the transfer 
rate on E1-E1 coupling between the donor and acceptor transition dipoles - a result of the inverse cubic dependence on their mutual separation $R$, in the near-field limit of the coupling tensor $\boldsymbol{V}$. However, in the chirally sensitive E1-M1 contribution to the rate, the near-field distance dependence runs with the inverse fifth power of the separation $R$, since $\boldsymbol{U}$ has an inverse square as its leading term [26, 27, 57]. Accordingly, the dissymmetry factor $g$ given by equation (5) has a perhaps surprising linear dependence on the donor-acceptor separation.

Similar features have long ago been flagged in connection with Raman optical activity, for example [88]: in that case it was subsequently shown that the result did not extend beyond the near-zone region $R<<\lambda / 2 \pi$, where $\lambda$ is the wavelength associated with the transfer energy $[89,90]$. In fact the same applies here; when the inter-chromophore distance $R$ is increased beyond the near-zone region, both $\boldsymbol{V}$ and $\boldsymbol{U}$ couplings acquire the same inverse power dependence, and so the distance dependence of $g$ will disappear. Nonetheless it is interesting to observe that, within the common range of Förster distances - generally much shorter than the near-zone limit, there is indeed a genuine increase with distance in the circular differential energy transfer rate. This may be considered to reflect the fact that, as a pair, an increase of separation within the donoracceptor couple develops increasing significance in relation to an electronic excitation wavelength. Still, it has to be emphasized that experimental exploitation of this feature is to some extent compromised by the fact that the absolute transfer rate does diminish with distance. Moreover, for any system in which the donor and corresponding acceptor units are orientationally uncorrelated, or freely mobile, the E1-M1 coupling - and hence the mechanism for chiral dissymmetry in which it is implicated - disappears (the mathematical result of rotational averaging). However, in most studies concerning the photophysics and photochemistry of proteins and other chiral biomolecules there is either very limited, or no such relative motion of the donor and acceptor moieties. This is a feature that is to be explored/in detail in forthcoming work on the full electrodynamic theory [91].

\section{Connections to other chiroptical effects}

As indicated earlier, there are other mechanisms by means of which circularly polarized light may be differentially engaged in RET and fluorescence processes. Those to be briefly discussed in this Section have not yet been experimentally identified - and it appears that most have not previously been considered. However, all are manifestly symmetry-allowed, and for these prospective effects directly analogous processes have been proven experimentally - so that there are compelling reasons to investigate the new possibilities.

It is illuminating to reflect on the connections that may exist between processes that are experimentally proven, and others which exist in an ostensibly time-inverse relation to 
them, in the sense discussed earlier, at the beginning of Section 3. As noted there, intramolecular vibrational relaxation will commonly intervene in any sequence of excitation and emission, especially in systems that have significantly different ground and excited state geometries; the effect is commonly reflected in a substantial Stokes shift [92]. Although for symmetry purposes the associated difference between absorption and emission wavelengths has no bearing on the conclusions over sign, the same cannot necessarily be concluded about the quantitative measures of circular dissymmetry.

Very recently, Tanaka et al. have thoroughly investigated the possibility of a correlation between the dissymmetry factors for circularly polarized luminescence and circular dichroism across a range of small organic molecules. They found a linear proportionality to exist between the two measures, within specific classes of compound, with a constant of proportionality that approached unity in the case of cyclophanes [93]. (Another broad correlation between CD and CPL is that both may occur in oriented samples that are not specifically chiral in 3D sense.)

Further insights may be afforded on recognition of connections between other chiroptical processes. For example, in the solution phenomenon known as induced circular dichroism (ICD), the absorption of light by an achiral molecule exhibits a circular intensity differential; the 'conferred chirality' arises as a result of the positioning of that molecule in the electronic environment of a neighbouring, chiral species [94]. Such a technique can indeed prove useful as a stereodynamic probe, proving particularly valuable in the study of protein interaction and binding $[95,96]$. At a fundamental level, the mechanism for ICD - which involves photon absorption by the achiral component coupled to a chiral species - is the time-inverse of a process in which the coupled achiral component emits a photon. (Of course, the use of a directed input beam in the specific case of ICD removes time-inverse connectivity at the multiparticle level). Both effects can be formally considered to arise from local modifications to the Maxwell fields near a chiral molecule [66, 97] - as indeed may also arise with plasmonic nanostructures [98-100] or within a chiral sculptured thin film [101], for example. The existence of induced CPL therefore reveals a propensity for achiral molecules in a chiral environment - such as may commonly occur in the solution phase when one component is the solvent and the other a solute - to exhibit circular differential fluorescence, even if initial excitation is achieved with plane polarized light.

The corresponding modified Feynman diagrams, shown in Fig. 6, entail E1 ${ }^{2}$ (polarizability, or transition polarizability) and E1M1 (gyration, or transition gyration) tensor interactions. In mechanism (a), for example, transfer of the optical excitation energy from the achiral molecule $\mathrm{A}$ to the chiral $\mathrm{C}$ has to immediately lead to emission through an interaction of essentially scattering form, since observation of the phenomenon depends on $\mathrm{C}$ lacking an excited state of the corresponding energy. Full 
details of the general theory for induced circularly polarized luminescence are provided in a foundational study by Salam [102].

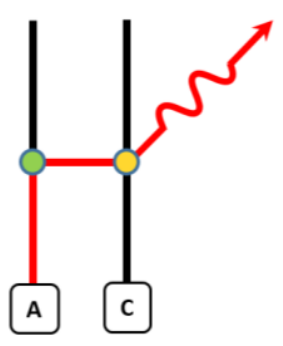

(a)

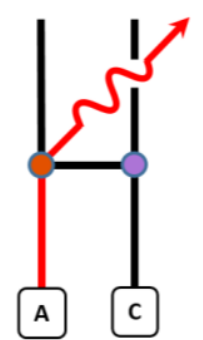

(b)

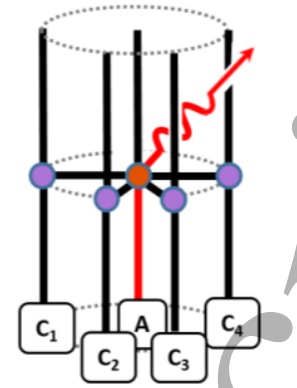

(c)

Figure 7. Modified Feynman diagrams for induced circularly polarized luminescence: time flows upwards, and the horizontal displacements between the vertical world-lines indicate a physical separation between participating fluorophores: (a) and (b) represent different contributions to the quantum amplitude for a process in which prior excitation (process not shown) of an achiral molecule $\mathrm{A}$, via coupling to a chiral neighbour $\mathrm{C}$, produces circular differential emission at the emission wavelength of $\mathrm{A}$; (c) portrays a similar effect, through mechanism (b), for an achiral molecule surrounded by chiral neighbours. Electronic excitation energy (which is shown flowing upwards along the red lines) emerges in the emitted photon. Interaction vertices are coloured green for transition electric dipole E1 coupling, yellow for transition tensors E1 ${ }^{2}$ or E1M1, red for transition $\mathrm{E} 1^{2}$ alone, and purple for static E1 or M1; other lines have the same meaning as in Fig. 4.

One further instance of the time-inverse relation is to be found in laser-assisted CPL. This is nominally a time-reversal of laser-assisted CD, in which an intense, circularly polarized beam essentially confers chirality upon an achiral system [103]. The original theory developed results for both circular dichroism and optical rotation; the latter effect has been identified in an auto-ionizing state of He [104]. CPL resulting from the throughput of an off-resonant circularly polarized beam, most effectively delivered during the lifetime of a previously excited achiral species, is therefore another process that is symmetry-allowed, and which indeed strongly relates to a general mechanism for the optical enhancement and suppression of fluorescence [105-107]. In view of recently discovered mechanisms to engage molecular chirality with optical vortex beams [53], a tantalizing question arises, as to whether the throughput of such a beam at a non-resonant frequency might also modify the extent of any circular differential in the luminescence from an excited chiral molecule. 


\section{Conclusion}

This review has identified a range of mechanisms and measurement strategies for circular differential fluorescence. As the analysis has shown, such an effect can always be anticipated to follow circular dichroic absorption, which is usually connected with single-photon absorption. In one recent and remarkable study of chiral conjugated polymers, it even appears that substantially different fluorescence decay pathways may be associated with optical inputs of opposite circularity, attributable to a degree of disorder in intermolecular interactions [108]. In this respect, it appears that chirality can be deployed as a probe of thermodynamically controlled disorder in supramolecular self-assembly.

Nonlinear optical forms of response represent a further tier of opportunities. Distinctive features have relatively recently been shown to arise with two-photon absorption in experiments on a variety of chiral organic systems [109-114], for which the corresponding CD was first predicted by theory many years ago [115-117] - and twophoton circular differential luminescence has indeed now been observed in helically arranged plasmonic nanoparticles [118]. As Friese leads the way in predicting a threephoton analogue of CD [119], it seems likely that proof of a CPL counterpart may also not be far away.

Reflecting further on the linear response that has been the main focus in this review, following the symmetry summary in Section 2, it is a significant feature of the tables provided in Section 3 that none of the extensive range of possibilities for permuting and combining optical and material handedness is excluded. Indeed, the electrodynamic theory that describes each series of interactions reveals a non-zero rate for every case. As was shown in Sections 4 and 5, the rate of energy transfer between chiral molecules has a subtle dependence on the handedness of each fluorophore - the energy donor and the acceptor. Moreover, the initial donor excitation will depend on the relative handedness of the input light and the donor; equally, resolving the FRET emission into circularly polarised components will manifest a difference whose sign is also influenced by the handedness of the acceptor.

Whilst it is true that marginal rate differences can arise in the wide variety of ways described, it is worth reiterating that there is no sense in which chirality can be said to transfer from one component to another. This is the clear message of the analysis in Section 6: there is no identifiable sense of helicity transfer, accompanying FRET. There is no electrodynamic mechanism, valid at the quantum level, to support the proposition. The closest analogue is spin-preserving energy transfer between exciton states in quantum dots - but these particles need not be chiral in the normal sense of the term.

[To clear a potential confusion, it should be pointed out that when coherent parametric optical processes are involved, (SHG - second harmonic generation - is a prominent 
example), the laws of angular momentum conservation apply since the input and output radiation are essentially collinear. The result is that certain combinations of handedness are indeed precluded; in fact, only by using circularly polarized beams with an optical vortex structure can the handedness of the input be unequivocally transmitted into the output - at the cost of one unit of orbital angular momentum.]

Section 8 described some more intricate mechanisms leading to circularly polarized emission, and it is interesting to consider the wider possibilities that now emerge, for the study of circular differential optical effects in propose-built surface nanostructures, especially those deposited from metals that can support local plasmonic enhancement. One of the remarkable opportunities that such studies can afford is the possibility to engineer a range of structures across which the sense of $2 \mathrm{D}$ chirality seamlessly changes sign [120] - a potentiality that almost never exists in the realm of molecular chemistry.

There are already wide-ranging applications of CPL, particularly in the sphere of biomolecules, many of them used to tackle issues of stereochemical configuration [121, 122]. In this connection, amino acids are frequently studied as ligands in lanthanide complexes [123-126], similar to the notional complex illustrated in Fig. 6(c). Here, the sharp $f \rightarrow f$ transitions of a central rare earth ion, providing a strong visible-region spectrum, provide an ideal basis for measurement of high $g$ value circularly polarized luminescence induced by coupling with the surrounding chiral amino acids. Such studies do not readily yield simple or comprehensive spectra-structure relationships, and even for the L/D nomenclature of amino acids tailored to the constituent functional groups, it has proved impossible to unequivocally base assignment on the sign of the CPL. Nonetheless, it is with the lanthanide complexes that some of the highest luminescence dissymmetry factors have been reported, as for example the value of 0.29 achieved in studying trivalent complexes of octadentate, 2-hydroxyisophthalamide ligands [127].

The breadth of application for sophisticated circular fluorescence measurements, in both direct and indirectemission processes, thus continues to expand in both diagnostic and device-oriented developments. As indicated in the report by Cerdán et al. [65], such advances are opening new avenues for developing cost-effective and easily processable chiro-photonic materials. The present review has indicated wide scope for many further advances in the spectroscopy of chiral materials, based on sound electrodynamic principles and informed by fundamental symmetry considerations.

\section{Acknowledgements}

The authors is pleased to acknowledge helpful comments from Dr David Bradshaw. I also thank my anonymous referees for several useful and thought-provoking suggestions. 


\section{References}

[1] Bakker H J and Skinner J L 2010 Vibrational Spectroscopy as a Probe of Structure and Dynamics in Liquid Water Chem. Rev. 110 1498-517

[2] Lin Y-S, Pieniazek P, Yang M and Skinner J 2010 On the calculation of rotational anisotropy decay, as measured by ultrafast polarization-resolved vibrational pump-probe experiments J. Chem. Phys. 132174505

[3] Mazur K, Heisler I A and Meech S R 2010 Ultrafast dynamics and hydírogenbond structure in aqueous solutions of model peptides The Journal of Physical Chemistry B 114 10684-91

[4] Barron L D 2004 Molecular Light Scattering and Optical Activity vol 2: Cambridge University Press Cambridge)

[5] Wenzel T J 2018 Prasad L. Polavarapu (Ed.): Chiral analysis: advances in spectroscopy, chromatography, and emerging methods Anal. Bioanal. Chem. $1-2$

[6] Canaguier-Durand A, Hutchison J A, Genet C and Ebbesen T W 2013 Mechanical separation of chiral dipoles by chiral light New J. Phys. 15123037

[7] Cameron R P, Barnett S M and Yao A M2014 Discriminatory optical force for chiral molecules New J. Phys. 16013020

[8] Bradshaw D S and Andrews D L 2014 Chiral discrimination in optical trapping and manipulation New J. Phys. 16103021

[9] Rukhlenko I D, Tepliakov N V, Baimuratov A S, Andronaki S A, Gun'ko Y K, Baranov A V and Fedorov A V 2016 Completely chiral optical force for enantioseparation Sci. Rep. 6

[10] Forbes K A and Andrews D L 2015 Chiral discrimination in optical binding Phys. Rev. A 91053824

[11] Bradshaw D S, Forbes K A, Leeder J M and Andrews D L 2015 Chirality in optical trapping and optical binding Photonics 2 483-97

[12] Bradshaw D S and Andrews D L 2015 Laser optical separation of chiral molecules Opt. Lett. 40 677-80

[13] Riehl J P and Richardson F S 1986 Circularly polarized luminescence spectroscopy Chem. Rev. 86 1-16

[14] Riehl J P and Richardson F S 1993 Circularly polarized luminescence Methods Enzymol. $226539-53$

[15] Clark R, Jeyes S, McCaffery A and Shatwell R 1974 Circular emission from molecular iodine Chem. Phys. Lett. 25 74-7

[16] Katô H, Clark R and McCaffery A 1976 Rotational assignments in excited iodine and reorientation by elastic and inelastic collisions from circularly polarized emission Mol. Phys. 31 943-56

[17] Clark R and McCaffery A 1978 Laser fluorescence studies of molecular iodine: I. Spectral assignments of the $5145 \AA$ Aluorescence Mol. Phys. 35 609-15 
[18] Rowe M and McCaffery A 1978 The influence of hyperfine coherence and of elastic collisions on the circular polarisation of emission from Li2 Chem. Phys. 34 81-94

[19] Bain A and McCaffery A 1984 Complete determination of the state multipoles of rotationally resolved polarized fluorescence using a single experimental geometry J. Chem. Phys. 80 5883-92

[20] Avery J 1966 Resonance energy transfer and spontaneous photon emission Proceedings of the Physical Society $\mathbf{8 8} 1$

[21] Gomberoff L and Power E 1966 The resonance transfer of excitation Proceedings of the Physical Society $\mathbf{8 8} 281$

[22] Andrews D L and Sherborne B S 1987 Resonant excitation transfer: A quantum electrodynamical study J. Chem. Phys. 86 4011-7

[23] Juzeliūnas. G and Andrews D L 1994 Quantum electrodynamics of resonant energy-transfer in condensed matter Phys. Rev. B 49 8751-63

[24] Scholes G D and Andrews D L 1997 Damping and higher multipole effects in the quantum electrodynamical model for electronic energy transfer in the condensed phase J. Chem. Phys. 107 5374-84

[25] Daniels G J, Jenkins R D, Bradshaw D S and Andrews D L 2003 Resonance energy transfer: The unified theory revisited J. Chem. Phys. 119 2264-74

[26] Andrews D L and Bradshaw D S 2004 Virtual photons, dipole fields and energy transfer: a quantum electrodynamical approach Eur. J. Phys. 25 845-58

[27] Salam A 2005 Resonant transfer of excitation between two molecules using Maxwell fields J. Chem. Phys. 122044113

[28] Novotny L and Hecht B 2006 Principles of Nano-Optics (Cambridge: Cambridge University Press)

[29] Andrews D L 2008 Mechanistic principles and applications of resonance energy transfer Can. J. Chem. 86 855-70

[30] Salam A 2010 Molecular Quantum Electrodynamics. Long-Range Intermolecular Interactions (Hoboken, NJ: Wiley)

[31] Longhi G, Castiglioni E, Koshoubu J, Mazzeo G and Abbate S 2016 Circularly polarized luminescence: a review of experimental and theoretical aspects Chirality 28 696-707

[32] Elemans J A, De Cat I, Xu H and De Feyter S 2009 Two-dimensional chirality at liquid-solid interfaces Chem. Soc. Rev. 38 722-36

[33] Kuwata-Gonokami M, Saito N, Ino Y, Kauranen M, Jefimovs K, Vallius T, Turunen J and Svirko Y 2005 Giant optical activity in quasi-two-dimensional planar nanostructures Phys. Rev. Lett. 95227401

[34] Zhang W, Potts A and Bagnall D 2006 Giant optical activity in dielectric planar metamaterials with two-dimensional chirality Journal of Optics A: Pure and Applied Optics $\mathbf{8} 878$

[35] Humblot V, Barlow S and Raval R 2004 Two-dimensional organisational chirality through supramolecular assembly of molecules at metal surfaces Prog. Surf. Sci. 76 1-19

[36] Brullot W, Vanbel M K, Swusten T and Verbiest T 2016 Resolving enantiomers using the optical angular momentum of twisted light Science Advances 2 e1501349 
[37] Ren H and Gu M 2018 Angular momentum-reversible near-unity bisignate circular dichroism Laser \& Photon. Rev. 121700255

[38] Andrews D L 2018 Quantum formulation for nanoscale optical and material chirality: symmetry issues, space and time parity, and observables J. Opt. 20 033003

[39] Andrews D L 2018 Symmetries, Conserved Properties, Tensor Representations, and Irreducible Forms in Molecular Quantum Electrodynamics Symmetry $\mathbf{1 0}$

[40] Harris A B, Kamien R D and Lubensky T C 1999 Molecular chirality and chiral parameters Rev. Mod. Phys. 71 1745-57

[41] Schäferling M, Yin X and Giessen H 2012 Formation of chiral fields in a symmetric environment Opt. Express 20 26326-36

[42] King R B 2003 Chirality and handedness Ann. N.Y. Acad. Sci. 988 158-70

[43] Petitjean M 2003 Chirality and symmetry measures: a transdisciplinary review Entropy 5 271-312

[44] Natarajan R and Basak S C 2009 Numerical characterization of molecular chirality of organic compounds Current Computer-Aided Drug Design 5 13-22

[45] Zhang Q-Y, Xu L-Z, Li J-Y, Zhang D-D, Long H-L, Leng J-Y and Xu L 2012 Methods of studies on quantitative structure-activity relationships for chiral compounds J. Chemom. 26 497-508

[46] Bancroft W D and Davis H L 1930 The Optical Rotation of Malic Acid The Journal of Physical Chemistry 34 897-928

[47] Guérin F and Lakhtakia A 1995 On the frequency-dependence of the chirality pseudoscalar of a chiral medium J. Phys. III 5 913-8

[48] Eyring H, Liu H-C and Caldwell D 1968 Optical rotatory dispersion and circular dichroism Chem. Rev. $68525-40$

[49] Bradshaw D S, Leeder J M, Coles M M and Andrews D L 2015 Signatures of material and optical chirality: Origins and measures Chem. Phys. Lett. 626 10610

[50] Smith G and Strange P 2018 Lipkin's conservation law in vacuum electromagnetic fields Journal of Physics A: Mathematical and Theoretical

[51] Andrews D L and Coles M M 2012 Measures of chirality and angular momentum in the electromagnetic field Opt. Lett. 37 3009-11

[52] Riehl J P and Muller G 2011 Circularly polarized luminescence spectroscopy and emission-detected circular dichroism Comprehensive Chiroptical Spectroscopy: Instrumentation, Methodologies, And Theoretical Simulations 1 65-90

[53] Forbes K A and Andrews D L 2018 Optical orbital angular momentum: twisted light and chirality Opt. Lett. $\mathbf{4 3}$ 435-8

[54] Salam A 2006 On the effect of a radiation field in modifying the intermolecular interaction between two chiral molecules J. Chem. Phys. 124014302

[55] Häse F, Roch L M, Kreisbeck C and Aspuru-Guzik A n 2018 Phoenics: A Bayesian Optimizer for Chemistry ACS Central Science

[56] Craig D P and Thirunamachandran T 1998 Molecular Quantum Electrodynamics: An Introduction to Radiation-Molecule Interactions (Mineola, NY: Dover Publications) 
[57] Rice E M, Bradshaw D S, Saadi K and Andrews D L 2012 Identifying the development in phase and amplitude of dipole and multipole radiation Eur. $J$. Phys. 33 345-58

[58] Salam A 2005 A general formula for the rate of resonant transfer of energy between two electric multipole moments of arbitrary order using molecular quantum electrodynamics J. Chem. Phys. 122044112

[59] Sakurai J J and Napolitano J 2017 Modern Quantum Mechanics (Cambridge: Cambridge University Press)

[60] Craig D P and Thirunamachandran T 1987 Change in chiroptical properties caused by intermolecular coupling Proc. R. Soc. Lond. A $410337-51$

[61] Craig D and Thirunamachandran T 1998 Chiral discrimination in molecular excitation transfer J. Chem. Phys. 109 1259-63

[62] Craig D and Thirunamachandran T 1999 New approaches to chiral discrimination in coupling between molecules Theor. Chem. Acc. 102 112-20

[63] Field J E, Muller G, Riehl J P and Venkataraman D 2003 Circularly polarized luminescence from bridged triarylamine helicenes J. Am. Chem. Soc. 125 11808-9

[64] Sánchez-Carnerero E M, Agarrabeitia A R, Moreno F, Maroto B L, Muller G, Ortiz M J and de la Moya S 2015 Circularly polarized luminescence from simple organic molecules Chem. Eur. J. 21 13488-500

[65] Cerdan L, Moreno F, Johnson M, Muller G, de la Moya S and Garcia-Moreno I 2017 Circularly polarized laser emission in optically active organic dye solutions Phys. Chem. Chem. Phys. 1922088-93

[66] Leeder J M, Haniewicz H T and Andrews D L 2015 Point source generation of chiral fields: measures of near-and far-field optical helicity J. Opt. Soc. Am. B 32 2308-13

[67] Andrews D L and Juzeliūnas. G 1991 The range dependence of fluorescence anisotropy in molecular-energy transfer J. Chem. Phys. 95 5513-8

[68] Leeder J M, Haniewicz H T and Andrews D L 2015 Surface functionalized spherical nanoparticles: an optical assessment of local chirality Proc. SPIE 954594502

[69] Selvin P R 2000 The renaissance of fluorescence resonance energy transfer Nat. Struct. Biol, 7 730-4

[70] Vogel S S, Nguyen T A, van der Meer B W and Blank P S 2012 The impact of heterogeneity and dark acceptor states on FRET: implications for using fluorescent protein donors and acceptors PloS one 7 e49593

[71] Masters T A, Marsh R J, Armoogum D A, Nicolaou N, Larijani B and Bain A J 2013 Restricted state selection in fluorescent protein Forster resonance energy transfer J. Am. Chem. Soc. 135 7883-90

[72] Blacker T S, Chen W, Avezov E, Marsh R J, Duchen M R, Kaminski C F and Bain A J 2017 Investigating state restriction in fluorescent protein FRET using time-resolved fluorescence and anisotropy The Journal of Physical Chemistry C 121 1507-14

[73] Duan P, Cao H, Zhang L and Liu M 2014 Gelation induced supramolecular chirality: chirality transfer, amplification and application Soft Matter 10 542848 
[74] Liu M, Zhang L and Wang T 2015 Supramolecular chirality in self-assembled systems Chem. Rev. 115 7304-97

[75] Yang D, Duan P, Zhang L and Liu M 2017 Chirality and energy transfer amplified circularly polarized luminescence in composite nanohelix Nat. Commun. 815727

[76] Jackson J D 1998 Classical Electrodynamics (New York: Wiley)

[77] Zangwill A 2013 Modern Electrodynamics (Cambridge: Cambridge University Press)

[78] Mandel L and Wolf E 1995 Optical Coherence and Quantum Optics (Cambridge, New York: Cambridge University Press)

[79] Scholes G D and Andrews D L 2005 Resonance energy transfer and quantum dots Phys. Rev. B 72125331

[80] Kroutvar M, Ducommun Y, Heiss D, Bichler M, Schuh D, Abstreiter G and Finley J J 2004 Optically programmable electron spin memory using semiconductor quantum dots Nature 43281

[81] Kruchinin S Y, Fedorov A, Baranov A, Perova T and Berwick K 2008 Resonant energy transfer in quantum dots: Frequency-domain luminescent spectroscopy Phys. Rev. B 78125311

[82] Guzelturk B, Martinez P L H, Zhang Q, Xiong Q, Sun H, Sun X W, Govorov A $\mathrm{O}$ and Demir H V 2014 Excitonics of semiconductor quantum dots and wires for lighting and displays Laser \& Photon. Rev. 8 73-93

[83] Andrews D L 2010 Optical angular momentum: Multipole transitions and photonics Phys. Rev. A $\mathbf{8 1} 033825$

[84] Bliokh K Y, Bekshaev A Y and Nori F 2013 Dual electromagnetism: helicity, spin, momentum and angular momentum New J. Phys. 15033026

[85] Nieto-Vesperinas M 2015 Optical theorem for the conservation of electromagnetic helicity: Significance for molecular energy transfer and enantiomeric discrimination by circular dichroism Phys. Rev. A 92023813

[86] Bene L, Bagdány M and Damjanovich L 2018 Checkpoint for helicity conservation in fluorescence at the nanoscale: Energy and helicity transfer (hFRET) from a rotating donor dipole Biophys. Chem. 239 38-53

[87] Grynberg G, Aspect A and Fabre C 2010 Introduction to Quantum Optics: From the Semi-Classical Approach to Quantized Light (Cambridge: Cambridge University Press)

[88] Barron L D and Buckingham A D 1974 Simple two-group model for Rayleigh and Raman optical activity J. Am. Chem. Soc. 96 4769-73

[89] Andrews D L and Thirunamachandran T 1978 A quantum electrodynamical theory of differential scattering based on a model with two chromophores. I. Differential Rayleigh-scattering of circularly polarized-light Proc. R. Soc. A $358297-310$

[90] Andrews D L and Thirunamachandran T 1978 A quantum electrodynamical theory of differential scattering based on a model with two chromophores. II. Differential Raman-scattering of circularly polarized-light Proc. R. Soc. A 358 $311-9$

[91] Bradshaw D S, Forbes K A and Andrews D L Work in progress 
[92] Andrews D L 2014 Molecular Photophysics and Spectroscopy (San Rafael, CA: Morgan \& Claypool Publishers)

[93] Tanaka H, Inoue Y and Mori T 2018 Circularly Polarized Luminescence and Circular Dichroisms in Small Organic Molecules: Correlation between Excitation and Emission Dissymmetry Factors ChemPhotoChem 2 386-402

[94] Craig D, Power E and Thirunamachandran T 1976 The dynamic terms in induced circular dichroism Proc. R. Soc. A 348 19-38

[95] Daviter T, Chmel N and Rodger A 2013 Protein-Ligand Interactions: Methods and Applications, ed M A Williams and T Daviter (New York: Humana Press) pp 211-41

[96] Wolf C and Bentley K W 2013 Chirality sensing using stereodynamic probes with distinct electronic circular dichroism output Chem. Soc. Rev. 42 5408-24

[97] Salam A and Thirunamachandran T 1994 Maxwell fields and Poynting vector in the proximity of a chiral molecule Phys. Rev. A 50 4755-66

[98] Hendry E, Mikhaylovskiy R V, Barron L D, Kadodwala M and Davis T J 2012 Chiral electromagnetic fields generated by arrays of nanoslits Nano Lett. 12 3640-4

[99] Abdulrahman N A, Fan Z, Tonooka T, Kelly S M, Gadegaard N, Hendry E, Govorov A O and Kadodwala M 2012 Induced Chirality through Electromagnetic Coupling between Chiral Molecular Layers and Plasmonic Nanostructures Nano Lett. 12 977-83

[100] Meinzer N, Hendry E and Barnes W L 2013 Probing the chiral nature of electromagnetic fields surrounding plasmonic nanostructures Phys. Rev. B 88 041407

[101] Mackay T G and Lakhtakia A 2007 Theory of light emission from a dipole source embedded in a chiral sculptured thin film Opt. Express 15 14689-703

[102] Salam A 1993 A quantum electrodynamical theory of induced circularly polarised luminescence Chem. Phys, 173 123-32

[103] Thirunamachandran T 1979 Theory of laser-induced optical activity Proc. $R$. Soc. A $365327-43$

[104] Gryzlova E, Grum-Grzhimailo A, Magunov A and Strakhova S 2008 Laser induced optical activity in the region of autoionizing states. In: Journal of Physics: Conference Series: IOP Publishing) p 012007

[105] Bradshaw D S and Andrews D L 2009 Mechanism for optical enhancement and suppression of fluorescence J. Phys. Chem. A 113 6537-9

[106] Bradshaw D S and Andrews D L 2010 All-optical control of molecular fluorescence Phys. Rev. A 81013424

[107] Leeder J M, Bradshaw D S and Andrews D L 2011 Laser-controlled fluorescence in two-level systems J. Phys. Chem. B 115 5227-33

[108] Sharma A, Athanasopoulos S, Tapping P C, Sabatini R P, McRae O F, Müllner M, Kee T W and Lakhwani G 2018 Emission decay pathways sensitive to circular polarization of excitation The Journal of Physical Chemistry C 122 23910-6

[109] Toro C, De Boni L, Lin N, Santoro F, Rizzo A and Hernandez F E 2010 TwoPhoton Absorption Circular Dichroism: A New Twist in Nonlinear Spectroscopy Chemistry-A European Journal 16 3504-9 
[110] Hernández F E and Rizzo A 2011 Two-photon polarization dependent spectroscopy in chirality: A novel experimental-Theoretical approach to study optically active systems Molecules 16 3315-37

[111] Loge C and Boesl U 2012 Laser mass spectrometry with circularly polarized light: two-photon circular dichroism Phys. Chem. Chem. Phys. 14 11981-9

[112] Savoini M, Wu X, Celebrano M, Ziegler J, Biagioni P, Meskers S C, Duò L, Hecht B and Finazzi M 2012 Circular dichroism probed by two-photon fluorescence microscopy in enantiopure chiral polyfluorene thin films J. Am. Chem. Soc. $1345832-5$

[113] Diaz C, Vesga Y, Echevarria L, Stará I G, Starỳ I, Anger E, Shen C, Moussa M E S, Vanthuyne N and Crassous J 2015 Two-photon absorption and two-photon circular dichroism of hexahelicene derivatives: a study of the effect of the nature of intramolecular charge transfer RSC Advances 5 17429-37

[114] Vesga Y and Hernandez F E 2017 Two-photon absorption and two-photon circular dichroism of 1-tryptophan in the near to far UV region Chem. Phys. Lett. 684 67-71

[115] Power E 1975 Two-photon circular dichroism J. Chem. Phys. 63 1348-50

[116] Tinoco Jr I 1975 Two-photon circular dichroism J. Chem. Phys. 62 1006-9

[117] Andrews D L 1976 A two-chromophore model for two-photon circulardichroism Chem. Phys. 16 419-24

[118] Singh H J, Saumitra, Singh V R, Sikdar S K, Jayaprakash B and Ghosh A 2016 Circular Differential Two-Photon Luminescence from Helically Arranged Plasmonic Nanoparticles ACS Photonics 3 863-8

[119] Friese D H and Ruud K 2016 Three-photon circular dichroism: towards a generalization of chiroptical non-linear light absorption Phys. Chem. Chem. Phys. 18 4174-84

[120] Collins J T, Zheng X, Braz N V, Slenders E, Zu S, Vandenbosch G A, Moshchalkov V V, Fang Z, Ameloot M and Warburton P A 2018 Enantiomorphing Chiral Plasmonic Nanostructures: A Counterintuitive Sign Reversal of the Nonlinear Circular Dichroism Advanced Optical Materials 1800153

[121] Gussakovsky E 2010 Reviews in Fluorescence 2008: Springer) pp 425-59

[122] McAlexander H R and Crawford T D 2015 Simulation of circularly polarized luminescence spectra using coupled cluster theory J. Chem. Phys. 142154101

[123] Muller G and Riehl J P 2005 Use of induced circularly polarized luminescence (CPL) from racemic D 3 lanthanide complexes to determine the absolute configuration of amino acids $J$ Fluoresc. 15 553-8

[124] Muller G 2009 Luminescent chiral lanthanide(III) complexes as potential molecular probes Dalton Trans. 9692-707

[125] Carr R, Evans N H and Parker D 2012 Lanthanide complexes as chiral probes exploiting circularly polarized luminescence Chem. Soc. Rev. 41 7673-86

[126] Zinna F and Di Bari L 2015 Lanthanide circularly polarized luminescence: bases and applications Chirality 27 1-13

[127] Petoud S, Muller G, Moore E G, Xu J, Sokolnicki J, Riehl J P, Le U N, Cohen S M and Raymond K N 2007 Brilliant Sm, Eu, Tb, and Dy chiral lanthanide 
complexes with strong circularly polarized luminescence J. Am. Chem. Soc.

$12977-83$ 